\title{
La educación para el desarrollo sostenible en el currículum universitario: una investigación-acción cooperativa con profesorado
}

\author{
Gisela Cebrián
}

\section{RESUMEN}

En este artículo se presenta una investigación-acción cooperativa, realizada en la Universidad de Southampton (Reino Unido), con el objetivo de promover el desarrollo curricular y profesional en educación para el desarrollo sostenible (EDS) y aprender de las prácticas cotidianas del personal académico. Se creó un grupo de trabajo interdisciplinario formado por cinco docentes universitarios de diferentes disciplinas (educación, arqueología, ingeniería informática, biología y ciencias de la salud) con el objetivo de apoyar la reflexión crítica y la acción del grupo para incorporar la EDS en su práctica docente. Se presentan los resultados en el desarrollo e innovación curricular, así como evidencias que muestran el impacto positivo del rol de facilitador. Tal y como muestran los resultados, este rol permitió la reflexión y la acción, junto con la identificación de las necesidades específicas del profesorado universitario participante y los factores que influyen en su participación y acción.

Palabras clave: educación para el desarrollo sostenible, investigación-acción, sostenibilidad, universidad, currículum, profesorado, aprendizaje activo, Reino Unido. 


\title{
A educação para o desenvolvimento sustentável no currículo universitário: uma pesquisa- ação cooperativa com professorado
}

\section{RESUMO}

Neste artigo se apresenta uma pesquisa-ação cooperativa, realizada na Universidade de Southampton (Reino Unido), com o objetivo de promover o desenvolvimento curricular e profissional em educação para o desenvolvimento sustentável (EDS) e aprender das práticas cotidianas do pessoal acadêmico. Se criou um grupo de trabalho interdisciplinar formado por cinco docentes universitários de diferentes disciplinas (educação, arqueologia, engenharia informática, biologia e ciências da saúde) com o objetivo de apoiar a reflexão crítica e a ação do grupo para incorporar a EDS na sua prática docente. Apresentam-se os resultados no desenvolvimento e inovação curricular, assim como evidencias que mostram o impacto positivo do trabalho de facilitador. Tal como mostram os resultados, este trabalho permitiu a reflexão e a ação, junto com a identificação das necessidades específicas do professorado universitário participante e dos fatores que influenciam em sua participação e ação.

Palavras chave: educação para o desenvolvimento sustentável, pesquisa-ação, sustentabilidade, universidade, currículo, professorado, aprendizagem ativa.

\section{Education for sustainable development in the university curriculum: cooperative action research with teachers}

\begin{abstract}
This article presents a case of cooperative action research, carried out at the University of Southampton (UK), with the aim of promoting curriculum and professional development in education for sustainable development (ESD) and learning from the day-to-day practices of academic staff. An interdisciplinary working group of five university teachers from different disciplines (Education, Archaeology, Computer engineering, Biology and Health sciences) was established to support the group's critical reflection and action to incorporate ESD into their teaching practice. Results in curriculum development and innovation are presented, as well as evidence showing the positive impact of the facilitator role. As the results show, this role allowed for reflection and action, along with the identification of the specific needs of the participating university faculty and the factors that influence their participation and action.
\end{abstract}

Key words: education for sustainable development, action-research, sustainability, university, curriculum, faculty, active learning.

Recepción: 11/01/18. Aprobación: 30/11/18. 


\section{Introducción}

Un gran número de universidades en todo el mundo han signado declaraciones nacionales e internacionales para impulsar la integración de la sostenibilidad en sus operaciones, en la gestión del campus y en el currículum (Alba, 2017; Lozano et al., 2013). La sostenibilidad en la educación superior (ES) es un área de investigación emergente (Wright, 2010), que hasta el momento se ha centrado principalmente en la gestión ambiental del campus y sus operaciones, en estudios de caso y ejemplos de buenas prácticas, y en la introducción de contenidos relacionados con la sostenibilidad en cursos específicos (Cebrián et al., 2015; Cotton et al., 2009; Fien, 2002). La gestión ambiental del campus ha visto más progreso que el desarrollo e innovación curricular para integrar los principios de la sostenibilidad (Jones et al., 2010), siendo poca la implementación y transformación holística de las universidades hacia los principios de la sostenibilidad (Cebrián et al., 2013; Lozano, 2006).

La sostenibilidad en la ES no es la simple adición de contenidos específicos sobre sostenibilidad (Sterling, 2004; Vilches y Gil, 2015), ya que implica un cambio cultural en las prácticas y estructuras educativas dominantes (Tilbury, 2012). El cambio hacia la sostenibilidad requiere enfoques integrales y sistémicos que conecten acciones en los diferentes ámbitos, como el curricular, los planes de estudios, el campus, la investigación y la comunidad universitaria (Müller-Christa et al., 2014). El desarrollo de una visión, misión y política universitaria clara en este ámbito, la creación e implementación de estrategias educativas e iniciativas de investigación en sostenibilidad, la transferencia y el trabajo conjunto entre los diferentes agentes de la sociedad son elementos clave para lograr universidades más sostenibles (Junyent $e t$ al., 2003; Velazquez et al., 2006).

Hasta ahora la mayor parte de la atención se ha puesto en la concreción de los enfoques pedagógicos necesarios para integrar la educación para el desarrollo sostenible (EDS) (Cotton et al., 2009). La EDS se refiere al "qué" y al "cómo", y a la promoción de metodologías de enseñanza-aprendizaje innovadoras (Ryan y Cotton, 2013). La integración de la EDS implica cambios en las estrategias pedagógicas actuales, en las que se debería pasar de: un aprendizaje basado en la transmisión a un aprendizaje basado en el descubrimiento y la experimentación; enfoques centrados en el profesorado a enfoques centrados en el alumnado, y de un aprendizaje teórico a un aprendizaje orientado a la acción que vincule teoría y práctica (Murga Menoyo, 2009; Sterling, 2004). La EDS se basa en la reflexión crítica y en el desarrollo de diferentes competencias como la capacidad de pensar en futuros alternativos, el pensamiento crítico, la participación activa, el trabajo colaborativo e interdisciplinario, y el pensamiento sistémico (Cebrián et al., 2014; Tilbury y Wortman, 2004). En este sentido, Tilbury (2007) ha señalado la utilización de enfoques basados en la acción como la investigación participativa, el aprendizaje activo o la investigaciónacción. Por otro lado, el aprendizaje transformativo para la sostenibilidad ha sido ampliamente discutido en la literatura como una pedagogía adecuada en materia de EDS (Sipos et al., 2008; Wals, 2010). Se basa en la integración de los dominios cognitivo, psicomotor y afectivo en el aprendizaje (Sipos et al., 2008). El objetivo es involucrar a los estudiantes en el cuestionamiento de sus propias cosmovisiones, creencias, sentimientos y valores, basados en experiencias vividas, para contribuir a la creación de nuevas perspectivas que tiendan a la sostenibilidad (Barrón et al., 2010; Wals, 2010). Del mismo modo, el aprendizaje basado en proyectos o problemas (ABP) y otras metodologías centradas en el alumnado, como el aprendizaje activo o el aprendizaje-servicio (AS), proporcionan oportunidades para trabajar desde el mundo real, fomentando la autorreflexión, la colaboración, la autonomía, la iniciativa y la resolución de problemas reales (Albareda-Tiana y GonzalvoCirac, 2013; Azcárate et al., 2012; Thomas, 2009). Se considera que el uso de este tipo de metodologías 
de enseñanza-aprendizaje centradas en el alumnado fomenta el desarrollo de las competencias necesarias para crear agentes de cambio hacia la sostenibilidad a través de la ES, profesionales empoderados capaces de hacer frente a la sostenibilidad mediante el pensamiento crítico y creativo (Barth et al., 2007; MurgaMenoyo, 2015).

Otras investigaciones recientes también han explorado los resultados de aprendizaje y competencias que los programas educativos de las universidades deberían desarrollar en los estudiantes, para que éstos se conviertan en agentes de cambio hacia la sostenibilidad (Aznar Minguet y Ull Solís, 2009; Cebrián et al., 2014; Wiek et al., 2011). Sin embargo, a pesar de la emergencia de este cuerpo de literatura, pocas evidencias existen sobre el desarrollo, los resultados y el impacto que estos cursos generan (Wiek et al., 2011). La participación del personal académico, sus conocimientos en sostenibilidad y su desarrollo profesional son fundamentales para una innovación curricular que permita el desarrollo de competencias en sostenibilidad (Bonil et al., 2012; Cebrián et al., 2014 y 2015; Lozano, 2006; Solís-Espallargas y Valderrama-Hernández, 2015). La investigación sobre el profesorado universitario se ha centrado principalmente en explorar sus conocimientos, actitudes, visiones y creencias en relación con la EDS (Cotton et al., 2009; Jones et al., 2008; Mora Penagos, 2012). En una investigación cualitativa centrada en explorar las opiniones del profesorado universitario sobre la EDS, Jones et al. (2008) identificaron como aspectos limitantes el interés personal, la falta de conciencia, de tiempo y capacidad. Otros inhibidores identificados en el desarrollo e innovación curricular hacia la sostenibilidad son: los académicos tienden a ver la sostenibilidad como una agenda impuesta y no conectada a su disciplina; la falta de conocimientos y competencias para integrar las cuestiones de sostenibilidad en sus asignaturas; el currículum oculto de las instituciones; la distribución por disciplinas, la falta de incentivos o de prioridad a nivel individual; el limitado compromiso y apoyo institucional; las demandas existentes del currículo establecido; el bajo compromiso de órganos externos implicados (Aznar et al., 2014; Cebrián et al., 2015; Leal Filho, 2009).

La investigación que se presenta en este artículo tuvo como objetivo reflejar y conectar las experiencias cotidianas y desafíos a los que se enfrentan los académicos en la práctica docente real, cuando tratan de integrar los principios de la EDS en su práctica docente, y cerrar de este modo la brecha identificada entre la teoría y la práctica en el campo de la EDS en la ES (Moore, 2005; Thomas, 2004).

Este estudio, concebido como una investigación-acción exploratoria, intentó contribuir a la identificación de las oportunidades, condiciones y estrategias para integrar la sostenibilidad, así como las contradicciones, retos y resistencias existentes al intentar modificar la práctica docente para integrar la EDS. A través de la creación de un grupo de trabajo de aprendizaje activo, formado por profesorado universitario proveniente de diferentes disciplinas, se pretendió promover un proceso de reflexión crítica grupal, para promover el desarrollo e innovación curricular en sostenibilidad.

\section{Contexto de la investigación}

Este proyecto de investigación se llevó a cabo en la Universidad de Southampton entre 2011 y 2014. Esta Universidad se encuentra en la región del Hampshire del Reino Unido; es una de las mejores universidades a nivel de investigación de esa región y una de las instituciones que conforman el Russell Group. La Universidad de Southampton cuenta con más de 22000 estudiantes y alrededor de 5000 miembros de personal académico. El perfil de la sostenibilidad en la Universidad se ha visto incrementado en los últimos años, hecho que evidencia el desarrollo de diferentes estrategias y planes relacionados con el medio ambiente y la sostenibilidad, como son la aprobación del segundo Plan de Gestión del Carbono en 2011, la Estrategia Ambiental y de 
Sostenibilidad en 2012 y la creación del Grupo Asesor en Medio Ambiente y Sostenibilidad en 2012.

En este trabajo se presentan los resultados de una tesis doctoral, en la que se llevó a cabo una investigación-acción, en la que la investigadora adoptó el rol de facilitadora, trabajando con un grupo de 5 profesores universitarios de diferentes disciplinas. La necesidad de colaborar y trabajar interdisciplinariamente (Jones et al., 2010; Tilbury, 2012), así como la existencia de diferentes percepciones y actitudes del profesorado ante la EDS (Cebrián et al., 2015; Cotton et al., 2009), llevó a la creación de este grupo de trabajo interdisciplinario y a la puesta en marcha de un proceso de investigación-acción que permitiera generar consciencia, nuevas prácticas y conocimientos, un aprendizaje transformativo y el empoderamiento del profesorado para llevar a cabo innovaciones curriculares para integrar la sostenibilidad (Wals, 2010). La concepción de interdisciplinariedad en este proyecto es patente en la formación del grupo colaborativo de cinco profesores universitarios de cinco disciplinas diferentes (biología, educación, ingeniería informática, ciencias de la salud y arqueología), que mediante sesiones de aprendizaje activo comparten e intercambian conocimientos y experiencias, y desarrollan visiones y prácticas compartidas en EDS.

\section{Método}

\section{El proyecto de investigación-acción}

Este proyecto se enmarca en un paradigma cualitativo, ya que el objetivo fue adquirir un conocimiento profundo del contexto de la Universidad de Southampton, y de las experiencias del profesorado, así como promover el desarrollo e innovación curricular (Bisquerra Alzina, 2004; Flick et al., 2004). La investigación-acción como método se centra en la mejora de problemas reales o situaciones a través de la participación, la colaboración, la acción y el empoderamiento de los participantes (Reason y Bradbury, 2008). La investigación-acción puede tomar diferentes formas como son la investigaciónacción participativa, la investigación-acción crítica, la investigación colaborativa y el aprendizaje activo (Kember y Associados, 2000; Latorre, 2007; Revans, 2011). Los principios metodológicos de la investigación-acción se basan en: la integración de la investigación y la acción; la creación de comunidades de aprendizaje y colaboración; un alto nivel de reflexividad; la estimulación del aprendizaje transformativo entre los participantes, y la inclusión de contextos políticos e históricos más amplios (Somekh, 2006). A su vez, la investigación-acción es un proceso cíclico de reflexión, planificación, acción y observación (McNiff y Whitehead, 2010).

Este estudio fue concebido como una investigación-acción cooperativa, ya que se basa en el trabajo conjunto de un grupo de profesorado, y en el cuestionamiento de las ideas y prácticas dominantes en materia de EDS a través de la reflexión crítica y la acción (Kemmis, 2010). Se pueden diferenciar tres dimensiones de la investigación-acción según su finalidad: personal, profesional y política; o según los agentes implicados: primera, segunda y tercera persona $(\mathrm{u}$ orden). De acuerdo con Reason y Bradbury (2008) la investigación-acción de segundo orden comienza con el diálogo interpersonal e incluye el desarrollo de comunidades de aprendizaje y organizaciones que aprenden. Así, se conformó un grupo de trabajo interdisciplinario de cinco académicos, de aprendizaje activo o aprendizaje en acción (Revans, 2011), generando espacios de discusión, colaboración, reflexión crítica y apoyo para el desarrollo de nuevos conocimientos y prácticas curriculares en EDS. En la tabla 1 se presenta el proyecto de manera resumida. 
Tabla 1. Resumen del proyecto

\begin{tabular}{|l|l|}
\hline Participantes & Cinco \\
\hline Género & Tres hombres y dos mujeres \\
\hline Áreas de conocimiento del profesorado & Educación, ingeniería informática, arqueología, biología y ciencias de la salud \\
\hline Roles dentro de la organización & Director de programa, profesor a tiempo completo y miembros del equipo de decanato. \\
\hline Métodos de recogida de datos & $\begin{array}{l}\text { Tres entrevistas individuales semiestructuradas con cada participante; tres sesiones en grupo de aprendizaje } \\
\text { activo. }\end{array}$ \\
\hline Muestreo & Muestreo intencional / Académicos con un interés previo en la sostenibilidad o EDS \\
\hline Criterios de selección de la muestra & $\begin{array}{l}\text { Académicos interesados en desarrollar la práctica docente e incorporar la EDS en sus materias; compartir } \\
\text { experiencias personales y profesionales; estar abierto al cuestionamiento de la propia práctica docente. }\end{array}$ \\
\hline
\end{tabular}

Fuente: Elaboración propia.

La investigadora adquirió el rol de facilitadora para el desarrollo e innovación curricular en EDS durante un periodo de un año y medio entre 2012 y 2013. Este rol incluyó la realización de entrevistas individuales, liderazgo y moderación de las sesiones de aprendizaje activo, preparación, intercambio y envío de documentos marco y recursos educativos en EDS, así como diferentes acciones de apoyo al profesorado (ver tabla 2). El proceso de análisis de datos cualitativos seguido fue el análisis temático (Rapley, 2011), que consistió en la transcripción de todas las entrevistas individuales y sesiones de aprendizaje activo, la codificación de la toda la información, la construcción de categorías y la búsqueda y establecimiento de patrones o temas emergentes en los datos analizados, mediante un proceso inductivo-deductivo (Braun y Clarke, 2006; Rapley, 2011).

Tabla 2. Proceso de investigación

\begin{tabular}{|l|l|}
\hline Acción & Breve descripción y objetivos \\
\hline Reunión informal & $\begin{array}{l}\text { Introducir a los participantes al proyecto de investigación. } \\
\text { Obtener consentimiento informado. } \\
\text { Los participantes aportaron ideas sobre asignaturas donde podrían introducir la sostenibilidad. }\end{array}$ \\
\hline $\begin{array}{l}\text { Envío de un documento sobre ideas clave } \\
\text { en EDS para el profesorado }\end{array}$ & $\begin{array}{l}\text { Compartir ideas teóricas y metodologías de enseñanza-aprendizaje sobre la EDS con los participantes. } \\
\text { Se preparó un documento con ideas clave para que los participantes pudieran revisar antes de la entrevista 1 y } \\
\text { tener así una discusión sobre las ideas en EDS de los participantes. }\end{array}$ \\
\hline Sesión de aprendizaje activo 1 (sAA1) & $\begin{array}{l}\text { Averiguar cuáles son los aspectos iniciales relacionados con la comprensión de la EDS y su práctica docente. } \\
\text { Explorar las ideas y comprensiones sobre la EDS por parte de los participantes, qué se está haciendo y qué se } \\
\text { podría hacer, así como la identificación de oportunidades, retos, herramientas y necesidades del profesorado. } \\
\text { Reflexionar sobre asignaturas donde se podría integrar la EDS. }\end{array}$ \\
\hline $\begin{array}{l}\text { Involucrar a los participantes en un grupo de discusión y reflexión crítica. } \\
\text { Cada participante trajo un desafío al que se enfrentan cuando se piensa en la EDS y en la integración en el } \\
\text { currículum. El grupo trabajó sobre los cinco desafíos presentados por los participantes. }\end{array}$ \\
\hline
\end{tabular}


La educación para el desarrollo sostenible en el currículum universitario...

\begin{tabular}{|c|c|}
\hline $\begin{array}{l}\text { Envío de un documento sobre recursos } \\
\text { educativos en EDS }\end{array}$ & $\begin{array}{l}\text { Proporcionar recursos educativos en EDS para apoyar la reflexión y el rediseño de la práctica docente para } \\
\text { integrar la sostenibilidad. Este documento contenía información general sobre recursos educativos en la ES y } \\
\text { específicos por área de conocimiento. }\end{array}$ \\
\hline $\begin{array}{l}\text { Envío de documento sobre temas } \\
\text { emergentes }\end{array}$ & $\begin{array}{l}\text { Compartir un documento resumen sobre los temas emergentes que surgieron en la entrevista } 1 \text { y SAA1. Este } \\
\text { documento se envió tres días antes de la entrevista } 2 \text { para poder reflexionar sobre los temas emergentes al inicio } \\
\text { de ésta. }\end{array}$ \\
\hline Entrevista 2 & $\begin{array}{l}\text { Averiguar el progreso o la falta de progresos realizados por cada participante. } \\
\text { Discusión sobre cómo los participantes habían progresado en relación con sus ideas iniciales, lo que había } \\
\text { sucedido desde la última reunión y dónde estaban a nivel de innovación curricular en ese momento; factores } \\
\text { influyentes en su progreso. }\end{array}$ \\
\hline $\begin{array}{l}\text { Envío de artículos sobre las actitudes } \\
\text { y percepciones de los estudiantes en } \\
\text { sostenibilidad }\end{array}$ & $\begin{array}{l}\text { Proporcionar recursos existentes e investigaciones sobre las actitudes y comprensiones de los alumnos en } \\
\text { sostenibilidad para apoyar su práctica docente. }\end{array}$ \\
\hline Roles dentro de la organización & $\begin{array}{l}\text { Introducir a los participantes al proyecto de investigación. } \\
\text { Obtener consentimiento informado. } \\
\text { Los participantes aportaron ideas sobre asignaturas donde podrían introducir la sostenibilidad. }\end{array}$ \\
\hline $\begin{array}{l}\text { Envío de un documento de trabajo sobre } \\
\text { la EDS }\end{array}$ & Recordar y refrescar a los participantes sobre la investigación, la EDS y elementos clave. \\
\hline Sesión de aprendizaje activo 2 (SAA2) & $\begin{array}{l}\text { Reflexionar y debatir las ideas contenidas en el documento de trabajo sobre la EDS. } \\
\text { Involucrar a los participantes en un grupo de discusión y la reflexión crítica sobre el diseño curricular para la } \\
\text { integración de la EDS. Los participantes reflexionaron en relación con los retos para el rediseño curricular en EDS. }\end{array}$ \\
\hline $\begin{array}{l}\text { Envío de documento sobre temas } \\
\text { emergentes }\end{array}$ & $\begin{array}{l}\text { Compartir un documento resumen sobre los temas emergentes que surgieron en la Entrevista } 2 \text { y SAA2. Este } \\
\text { documento se envió tres días antes de la entrevista } 3 \text { para poder reflexionar sobre los temas emergentes al inicio } \\
\text { de ésta. }\end{array}$ \\
\hline Entrevista 3 & $\begin{array}{l}\text { Discusión sobre todo el proceso y el rediseño de las asignaturas. Identificar los factores clave que han permitido } \\
\text { o limitado el rediseño curricular. } \\
\text { Reflexionar sobre el proceso de cambio, los posibles resultados y los elementos claves, estrategias y condiciones. }\end{array}$ \\
\hline Sesión de aprendizaje activo 3 (SAA3) & $\begin{array}{l}\text { Reflexionar sobre el proceso de investigación, el impacto y los resultados individuales y comunes. Condiciones } \\
\text { personales, profesionales y organizacionales para integrar la sostenibilidad en el currículum y práctica docente. }\end{array}$ \\
\hline
\end{tabular}

Fuente: Elaboración propia.

\section{Técnicas utilizadas}

\section{Entrevistas semiestructuradas}

La entrevista semiestructurada fue elegida porque permitía adquirir una comprensión profunda del problema de investigación y del mundo a través de la comprensión de las diferentes perspectivas y experiencias de los participantes (Bisquerra Alzina, 2004; Kvale y Brinkmann, 2009). Este tipo de técnicas cualitativas permiten una mayor flexibilidad y la posibilidad de generar un ambiente más confortable, donde se puede desarrollar una relación de confianza entre el investigador y los participantes, y donde pueden emerger puntos de vista relacionados con la sostenibilidad. Las entrevistas fueron diseñadas según temas generales de discusión, utilizando preguntas abiertas, que permitían a los participantes hablar en profundidad sobre cuestiones relacionadas con la EDS y su desarrollo e innovación curricular que eran importantes y relevantes para ellos.

\section{Sesiones de aprendizaje activo}

El aprendizaje activo es un proceso de reflexión y aprendizaje incesante que se lleva a cabo con el apoyo de un grupo de compañeros, con el objetivo de 
tomar acciones para mejorar la práctica real (McGill y Brockbank, 2004). En este proyecto el aprendizaje activo fue concebido como un enfoque de enseñanza-aprendizaje para la EDS y una técnica de recogida de datos. La creación de un grupo de trabajo basado en el aprendizaje activo fue utilizada para promover la reflexión crítica, el aprendizaje y el desarrollo de nuevos conocimientos y prácticas en EDS entre los participantes (Revans, 2011). La reflexión y la acción se sitúan en el centro del proceso de aprendizaje activo, ya que promueven la reflexión sobre las experiencias previas, permiten repensar la práctica actual, así como el desarrollo de nuevos conocimientos entre los miembros del grupo de aprendizaje activo (McGill y Brockbank, 2004). Se pidió a los participantes que trajeran un desafío o reto en cada una de las tres sesiones, centrándose en las experiencias personales y profesionales previas, y el marco de la EDS en la primera sesión; en el diseño e innovación curricular en la segunda sesión, y en las condiciones personales, profesionales y de la organización para integrar al EDS en la última sesión. Esto permitió obtener información sobre el proceso individual y grupal de desarrollo e innovación curricular. También se identificaron oportunidades y retos en el proceso de integración de la EDS en el currículum. En esta investigación cada una de las sesiones de aprendizaje activo siguió la estructura de conversaciones de aprendizaje activo, que se basan en un proceso cíclico repetido para cada desafio o reto presentado por los participantes (Marsick y Maltbia, 2009):

- Plantear el desafío/reto como una cuestión

- Descifrar el significado del desafío/reto a través de intercambio de información sobre el contexto y la acción previa

- Cuestionamiento por pares (al que el titular del problema no responde de inmediato) para desbloquear los modelos mentales que hacen que uno no tenga en consideración otros puntos de vista
- Identificar los supuestos que subyacen en las formas actuales de enmarcar/entender el reto

- Replantear la propia comprensión de la situación

- Tomar decisiones y acciones informadas para abordar el reto

Las conversaciones de aprendizaje activo difieren de otros enfoques de aprendizaje activo. Éstas ponen énfasis en la reflexión crítica sobre los posibles supuestos de los miembros del grupo en el momento de plantear el desafio, o al pensar en nuevas prácticas y acciones futuras. Este enfoque fue escogido debido al interés de la investigadora en la promoción de la reflexión crítica y el cuestionamiento de asunciones, valores y creencias en EDS entre los participantes (Kemmis, 2010).

\section{Resultados del proyecto de investigación-acción Oportunidades y retos para la integración de las EDS en el currículum}

Los hallazgos de esta investigación reflejan la dificultad del profesorado universitario para comprender la naturaleza holística de la sostenibilidad, así como para entender la sostenibilidad como un proceso de aprendizaje en lugar de una lista de verificación (Tilbury, 2012).

El desafío es su naturaleza holística, ya que es en parte un entorno y una geografía, ¿no es así? y la geología, es en parte económica, es parte de la política, es parte de la cultura, interactúa con muchas áreas diferentes $[. .$.$] te obliga a ir a lugares donde no$ has estado (Participante 1).

Sin embargo, las diferentes perspectivas sobre la sostenibilidad también fueron vistas como un aspecto estimulante. Por ejemplo, la participante 4 en la entrevista 2 hizo referencia al documento resumen con los temas emergentes de la SAAl, y enfatizó "creo que lo más interesante fue cuando leí lo de todo el 
mundo [...] el documento resumen que enviaste y es bastante interesante cómo se puede entender la sostenibilidad desde diferentes perspectivas". Los participantes reconocieron una mayor conciencia de la sostenibilidad entre la comunidad académica, sin embargo, consideraron también que algunos colegas no veían la relevancia o beneficios. De acuerdo con el participante 3 en la entrevista 2, "el profesorado no ve la relación o no piensan que sea algo relevante en su práctica docente".

Estos resultados indican que un paso previo a la integración de la EDS en el currículum es dotar al profesorado de un conocimiento previo y una comprensión profundos de la sostenibilidad, y conocer la relevancia que ésta tiene sobre la práctica docente y su disciplina. Estos hallazgos refuerzan los resultados obtenidos por estudios previos (Christie et al., 2013; Cotton et al., 2009) en que los que se pone de manifiesto que parte del profesorado universitario no ve el vínculo entre la sostenibilidad y su disciplina o área académica. Es necesario crear estrategias y procesos de aprendizaje que permitan cuestionar los modelos mentales existentes en relación con la EDS, para generar un aprendizaje transformativo (Senge, 2006; Sterling, 2004). Brindar oportunidades de capacitación y desarrollo profesional al profesorado universitario que puedan permitir desarrollar nuevas comprensiones y conocimientos, son acciones necesarias para involucrar a los académicos e integrar la EDS en el currículo (Holdsworth et al., 2007).

La sostenibilidad, vista como un área de investigación de actual relevancia, los intereses de los estudiantes y la posición y el rol de los académicos dentro de la universidad, permitieron integrar la EDS en su enseñanza actual.

Es un área de investigación realmente importante, por lo que es bueno para [los estudiantes] ver que ésta es un área en crecimiento y también debería ser muy emocionante para ellos estar investigando algo que es tan nuevo (Participante 4-Entrevista 1).
La falta de tiempo, los recursos financieros y los recursos materiales para la enseñanza se destacaron como desafíos para involucrarse en la EDS.

Es el tiempo, éste es el problema. Todo el mundo está bajo presión, tienen muchas cosas que tienen que lograr y tienen que hacer, y la mayoría de las personas tienen dos o tres roles y responsabilidades diferentes (Participante 2-entrevista 2).

La conciencia y el conocimiento de los recursos de enseñanza existentes podrían aumentar la motivación de los académicos para integrar la EDS en su práctica docente: "Algunos estudios de caso útiles serían geniales" (Participante 3-entrevista 1).

Existían diferentes puntos de vista sobre la enseñanza de la sostenibilidad y la necesidad de integrarla en el currículum. Los participantes valoraron las metodologías de enseñanza-aprendizaje de la EDS; sin embargo, surgieron una serie de desafíos en la práctica real, como las expectativas de los estudiantes, el currículum basado en evaluaciones y la existencia de un currículum ya concurrido con diferentes demandas. "Creo que suponemos que, si no lo evaluamos, los estudiantes no lo valoran" (Participante 5-SAA1).

Los hallazgos muestran que la contextualización de la sostenibilidad de acuerdo con el área temática y la búsqueda de un enfoque específico son oportunidades para integrar la EDS en el currículum. Es necesario encontrar el lugar adecuado para integrarla en cada área temática (Ryan y Cotton, 2013). Los resultados de esta investigación muestran que el uso de la pedagogía de la EDS por parte del profesorado universitario está condicionado por sus valores y creencias previas existentes en relación con los procesos de enseñanza y aprendizaje, y por la naturaleza de su área temática (Cotton et al., 2009).

Quiero decir que tú obviamente crees en la importancia del dominio afectivo en el aprendizaje. Ésta no es mi tipo de mentalidad histórica [...] yo 
soy un positivista, hay verdades, que la función del aprendizaje es desarrollar a las personas cognitivamente [...] en un sentido, sus emociones seguirán su cerebro, el corazón sigue a la cabeza (Participante 1-entrevista 1).

Además, los procesos de evaluación externa y de garantía de calidad se consideraron como cruciales para integrar la EDS en el currículo. "Mi modelo es sobre los procesos de garantía de calidad, utilizando éstos para garantizar que exista un requisito para que hagas esto" (Participante 3-entrevista 3).

La comunicación, el apoyo de la organización y el liderazgo por parte del equipo rectoral emergieron como oportunidades para el compromiso del profesorado en la EDS. El panorama cambiante de la educación superior ofreció a los académicos la oportunidad de crear nuevas formas de enseñanza. La cultura académica y las presiones de investigación se destacaron como desafíos para integrar la EDS.
No encaja en el tipo de estructuras académicas en las que tenemos nuestras vidas inmersas, por lo que es un campo difícil de integrar en la educación superior (Participante 1-SAA2).

\section{Resultados con relación al desarrollo e innovación curricular en EDS}

En esta sección se presentan los principales resultados alcanzados por los cinco participantes durante el proceso de investigación a nivel de desarrollo e innovación curricular, así como evidencias de aprendizaje individual y colaborativo hacia la sostenibilidad. Los participantes consiguieron algunos resultados plausibles durante el proceso de investigación-acción. En la figura 1 se muestran gráficamente las diferentes innovaciones y acciones llevadas a cabo por los cinco académicos participantes, que se han situado en relación con el ciclo de investigación-acción: planificación, implementación, observación y evaluación. De este modo se sitúan los participantes en relación con la fase a la que llegaron durante el tiempo de duración del proyecto.

\section{Figura 1. Innovaciones curriculares de los participantes}

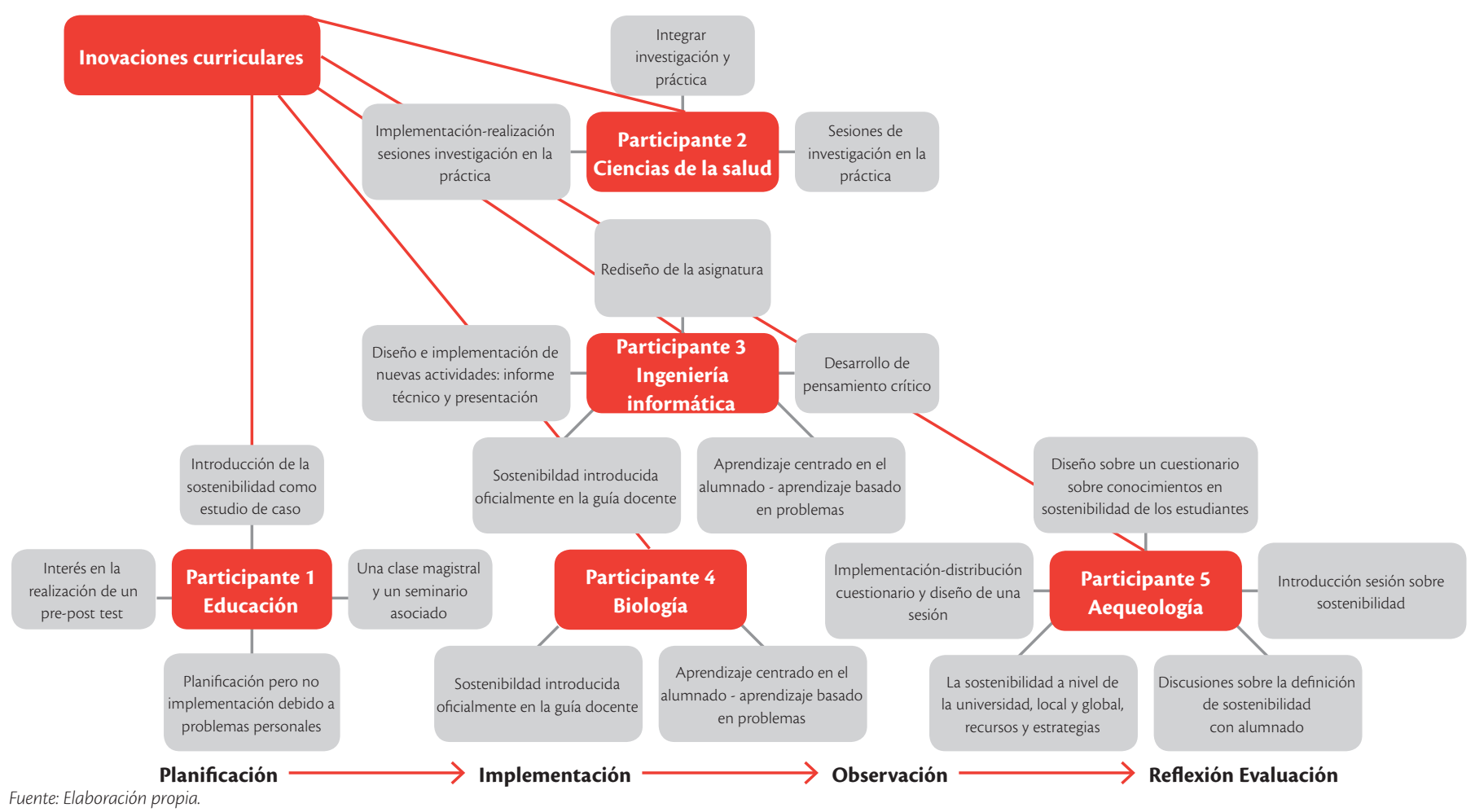


El proceso de investigación y la participación en un grupo de trabajo interdisciplinario y de aprendizaje activo motivó a los participantes en la integración de la sostenibilidad en el currículo, llevando a la introducción oficial de la sostenibilidad en los planes de estudio y guías docentes. Por ejemplo, el participante 3 , proveniente de ingeniería informática, introdujo la sostenibilidad en la guía docente y en la evaluación. También creó e implementó dos nuevas actividades relacionadas con la sostenibilidad: la producción de un informe técnico y la realización de una presentación oral relacionada con la sostenibilidad.

El curso ahora tiene una serie de lugares en los que los alumnos están estudiando algunos aspectos de la sostenibilidad [...] les dimos a los alumnos la opción de seis títulos de informes técnicos, tenían que elegir uno de ellos, y cuatro de ellos estaban relacionados con la sostenibilidad [...] y de manera similar a la hora de hacer presentaciones [...] creo que había tal vez dos de los doce temas (Participante 3).

No se pudieron recoger evidencias claras sobre el impacto que la investigación tuvo en el rediseño de la asignatura e inclusión de nuevas actividades por parte del participante 3 ya que, debido a su interés personal, él tenía previamente la intención de integrar la sostenibilidad. Sin embargo, reconoció que el proyecto había reforzado sus ideas iniciales, considerando que: "El proyecto ha sido interesante, pero habríamos hecho esto [...] esto iba a suceder y, en cierta medida ya estaba sucediendo, así que tal vez lo que el proyecto hizo fue hacernos reforzarlo, poner más énfasis en ello" (Participante 3).

El participante 1 proveniente del área de educación, planificó introducir la sostenibilidad como un estudio de caso, otorgándole una clase magistral y un seminario, aunque debido a motivos personales finalmente no pudo realizar estas sesiones, y por este motivo se le sitúa en la fase de planificación.
La participante 4 diseñó un cuestionario para obtener información sobre los conocimientos previos de los estudiantes en sostenibilidad. La idea de preparar un pre-post-test con los estudiantes surgió durante una de las sesiones de aprendizaje activo. De este modo, los resultados obtenidos en el pre-test sirvieron para diseñar una sesión con el alumnado, en la que se vieron las diferentes definiciones de sostenibilidad, recursos para prácticas diarias sostenibles, así como la sostenibilidad a diferentes niveles, como en la propia universidad, la comunidad local y a nivel global. Los resultados del cuestionario fortalecieron su motivación y facilitaron la introducción de la sostenibilidad en su asignatura. La participante 4 hizo hincapié en que:

Uno de los desafíos era pensar sobre cómo introducir algo nuevo y exactamente cómo hacerlo y pensar en qué iba a ser introducido, de manera que [la facilitadora] y yo desarrollamos este cuestionario juntas para averiguar qué es lo que los estudiantes ya saben, y se obtuvieron resultados bastante interesantes a través del cuestionario y fui capaz de mostrar a los estudiantes algunos de estos resultados como un seguimiento, así que me decidí a hacer una sesión sobre sostenibilidad basada en los resultados del cuestionario y luego volví a pasar el cuestionario (Participante 4).

Al finalizar la sesión sobre sostenibilidad se volvió a distribuir el cuestionario con el fin de poder comparar los conocimientos de los estudiantes antes y después de la intervención, y así reunir pruebas sobre el impacto en el aprendizaje de los estudiantes.

El participante 5, proveniente de arqueología, ya estaba introduciendo la sostenibilidad en sus asignaturas. Arqueología es un área de conocimiento de naturaleza multidisciplinar. Su participación en el proyecto contribuyó a la reflexión sobre el contenido y desarrollo del currículum desde una perspectiva de la EDS, teniendo un impacto en sus ideas sobre la 
aplicación práctica del plan de estudios. El participante 5 reconoció que el proyecto había fomentado un cambio de aproximación sobre la sostenibilidad, de una aproximación más descriptiva y analítica a una más proactiva sobre la enseñanza de la sostenibilidad; comentó que:

En relación con la transmisión del qué y el por qué, y teniendo en cuenta lo que está incluido y lo que no está incluido ha cambiado debido a la reflexión y pensamiento sobre este concepto de educación para la sostenibilidad $[. .$.$] he puesto un propósito un poco$ diferente, de uno de descripción y presentación de informes y análisis, a uno que ha pasado a una agenda mucho más proactiva al respecto [...] realmente es bueno pensar en ello y bueno para darle un empujón (Participante 5).

La participante 2 decidió centrarse en una serie de sesiones con los estudiantes a las que nombró "investigación-en-práctica". Estas sesiones contribuyeron a que los estudiantes desarrollaran habilidades de investigación, vinculándolas con la práctica diaria de los profesionales. Esta innovación correspondía a la preparación y desarrollo de tres sesiones para los estudiantes de primer curso, que se centraron en la lectura y debate crítico de trabajos de investigación útiles para la futura práctica profesional de los estudiantes. Para los estudiantes de segundo curso se diseñaron tres sesiones centradas en la investigación cualitativa, cuantitativa y método mixto respectivamente, que ayudaban a los estudiantes con sus trabajos de fin de grado. La participante 2 explicó que:

Para el nuevo plan de estudios fue la creación de las sesiones de investigación-en-práctica y clubes de revistas científicas, para el primer y segundo año, de modo que pudieran tener las habilidades que les ayuden al aprendizaje a lo largo de la vida y ser sostenibles en su rol profesional (Participante 2).
Si nos fijamos en las principales fases de un ciclo de investigación-acción típico: planificación, implementación de la acción, observación, y reflexiónevaluación (McNiff y Whitehead, 2010), tal y como se puede observar en la figura 1 , existieron diferencias entre los participantes. Los participantes 2, 3 y 4 llegaron a implementar innovaciones curriculares, aunque no se pudieron recopilar evidencias para evaluar si llegaron o no a la fase de reflexión/ evaluación, ni de cómo este primer ciclo informó al siguiente ciclo de investigación-acción, ni acerca de su práctica docente el siguiente curso académico. El participante 1 planeó una semana para introducir un estudio de caso sobre sostenibilidad sin embargo no pudo llevarla a cabo. Los participantes 2, 3 y 4 diseñaron nuevos contenidos y estrategias docentes para integrar la sostenibilidad, introduciéndola como una sesión o actividades específicas.

La sostenibilidad fue principalmente introducida como contenido específico, estudio de caso, o clases magistrales sobre la temática, resultados que distan del objetivo de la EDS de integrar la sostenibilidad de forma holística y transversal en el currículum (Sterling, 2004; Tilbury, 2012). Tal y como señalan otros estudios (Jones et al., 2008; Leal Filho, 2009) existen diferentes factores que condicionan la integración de la sostenibilidad en el currículum como: las demandas y requerimientos existentes de las diferentes titulaciones, la falta de tiempo, incentivos y conocimiento profundo sobre la sostenibilidad. Cabe destacar que los participantes adquirieron nuevos conocimientos en sostenibilidad. Las sesiones de aprendizaje activo, las reuniones de trabajo colaborativo, el rol de la facilitadora, los recursos educativos compartidos con los participantes, así como el apoyo recibido durante el proyecto, contribuyeron a un aumento de la motivación del profesorado, reforzando su trabajo previo y motivando la introducción de nuevos elementos relacionados con la EDS en su práctica docente. 


\section{Conclusiones}

El método de investigación-acción utilizado, juntamente con las sesiones de aprendizaje activo y las acciones llevadas a cabo por la facilitadora, permitieron la reflexión crítica sobre las concepciones previas del profesorado universitario en relación con la sostenibilidad, y contribuyeron al desarrollo de nuevos conocimientos, perspectivas y prácticas en materia de EDS. La investigación-acción y el aprendizaje activo son instrumentos adecuados para trabajar con el profesorado en el desarrollo profesional y la innovación curricular. Estos enfoques proporcionan espacios de reflexión y colaboración entre el profesorado universitario, dotándoles de tiempo para reflexionar conjuntamente y de manera crítica sobre la práctica de la EDS. Este espacio de reflexión y diálogo promueve el desarrollo de nuevas prácticas docentes, debido a la adquisición de nuevos conocimientos y puntos de vista sobre la EDS. Por este motivo se considera que es necesario llevar a cabo más investigaciones empíricas que utilicen la investigación-acción y técnicas participativas para fomentar nuevas perspectivas y el desarrollo profesional y curricular en EDS. Este tipo de proyectos se basan en la mejora de un problema que parte de la práctica docente real y se centra en el proceso. Investigaciones de este tipo pueden informar sobre nuevas prácticas docentes y futuros estudios sobre los métodos y estrategias más adecuados para trabajar con el profesorado universitario, sobre cómo cambiar el pensamiento y la práctica docente en EDS.

Para involucrar al profesorado universitario en la EDS es preciso crear programas de desarrollo profesional y formación permanente, grupos de trabajo colaborativo e interdisciplinarios basados en la investigación y la acción, que puedan informar futuras propuestas de innovación curricular en este ámbito. A su vez, el apoyo y liderazgo institucional debe crear una comunidad de aprendizaje donde se identifiquen buenas prácticas existentes, se promueva el intercambio de recursos educativos y se disponga de apoyo y orientación por parte de expertos y facilitadores. Para asegurar la sostenibilidad de proyectos de este tipo es indispensable proporcionar al profesorado académico el tiempo y los recursos económicos suficientes, que le permitan trabajar a largo plazo para el rediseño del currículo, la integración de la sostenibilidad y otros aspectos que deben ser integrados transversalmente en el currículo. 


\section{Referencias}

Alba Hidalgo, D. (2017), "Hacia una fundamentación de la sostenibilidad en la educación superior", Revista Iberoamericana de Educación, núm. 73, pp. 15-34.

Albareda-Tiana, S. y M. Gonzalvo-Cirac (2013), "Competencias genéricas en sostenibilidad en la educación superior. Revisión y compilación", Revista de Comunicación de la SEECI, núm. 32, pp. 141-159.

Azcárate, P., A. Navarrete y E. García (2012), "Aproximación al nivel de inclusión de la sostenibilidad en los currícula universitarios", Profesorado. Revista de Currículum y Formación del Profesorado, vol. 16, núm. 2, pp. 105-1 19.

Aznar Minguet, P. y M. A. Ull Solís (2009), "La formación de competencias básicas para el desarrollo sostenible: el papel de la Universidad", Revista de Educación, núm. Extraordinario, pp. 219-237.

Aznar, P., M. A. Ull, A. Piñero y M. P. Martínez-Agut (2014), "La sostenibilidad en la formación universitaria: Desafíos y oportunidades", Educación XXI, vol. 17, núm. 1, pp. 133-158.

Barrón, A., A. Navarrete y D. Ferrer-Balas (2010), "Sostenibilización curricular en las universidades españolas. ¿Ha llegado la hora de actuar?”, Revista Eureka sobre Enseñanza y Divulgación de las Ciencias, vol. 7, núm. 1, pp. 388-399.

Barth, M., J. Godemann, M. Rieckmann y U. Stoltenberg (2007), "Developing key competencies for sustainable development in higher education", International fournal of Sustainability in Higher Education, vol. 8, núm. 4, pp. 416-430.

Bisquerra Alzina, R. (2004), Metodología de la investigación educativa, Madrid, La Muralla.

Bonil, J., G. Calafell, J. Granados Sánchez, M. Junyent y R. M. Tarín (2012), "Un modelo formativo para avanzar en la ambientalización curricular", Profesorado. Revista de Currículum y Formación de Profesorado, vol. 16 núm. 2, pp. 145-163.

Braun, V. y V. Clarke (2006), "Using thematic analysis in psychology", Qualitative Research in Psychology, núm. 3, pp. 77-101.
Cebrián, G., M. Grace y D. Humphris (2015), "Academic staff engagement in education for sustainable development", fournal of Cleaner Production, 106, pp. 79-86.

Cebrián, G. y M. Junyent (2014), “Competencias profesionales en educación para la sostenibilidad: un estudio exploratorio de la visión de futuros maestros", Enseñanza de las Ciencias, 32(1), pp. 29-49.

Cebrián, G., M. Grace y D. Humphris (2013), "Organisational learning towards sustainability in higher education", Sustainability Accounting, Management and Policy Fournal, 4(3), pp. 285-306.

Christie, B. A., K. K. Miller, R. Cooke y J. G. White (2013), "Environmental sustainability in higher education: how do academics teach?", Environmental Education Research, vol. 19, núm. 3, pp. 385-414.

Cotton, D., I. Bailey, M. Warren y S. Bissell (2009), "Revolutions and second-best solutions: education for sustainable development in higher education", Studies in Higher Education, vol. 34, núm. 7, pp. 719-733.

Fien, J. (2002), "Advancing sustainability in higher education. Issues and opportunities for research", International Fournal of Sustainability in Higher Education, vol. 3, núm. 3, pp. 243-253.

Flick, U., E. von Kardorff e I. Steinke (eds.) (2004), A companion to qualitative research, Londres, SAGE Publications Ltd.

Holdsworth, S., C. Wyborn, S. A. Bekessy e I. Thomas (2007), "Professional development for education for sustainability: How advanced are Australian universities?", International Fournal of Sustainability in Higher Education, vol. 9, núm. 2, pp. 131-146.

Jones, P., D. Selby y S. Sterling (2010), "Introduction”, en Sustainability education: perspectives and practice across higher education, Londres, Earthscan, pp. 1-16.

Jones, P., C. J. Trier y J. P. Richards (2008), "Embedding education for sustainable development in higher education: a case study examining common challenges and opportunities for undergraduate programmes", 
International fournal of Educational Research, vol. 47, núm. 6, pp. 341-350.

Junyent, M., A. M. Geli y E. Arbat (coords.) (2003), Ambientalización curricular de los estudios superiores. 2. Proceso de caracterización de la ambientalización curricular de los estudios universitarios, Girona, Red ACES/Universitat de Girona.

Kember, D. y asociados (2000), Action learning and action research: improving the quality of teaching and learning, Londres, Kogan Page.

Kemmis, S. (2010), "What is to be done? The place of action research", Educational Action Research, vol. 18 núm. 4, pp. 417-427.

Kvale, S. y S. Brinkmann (2009), Interviewes: learning the craft of qualitative research interviewing ( $2^{\mathrm{a}} \mathrm{ed}$.), Thousand Oaks, SAGE Publications.

Latorre, A. (2007), La investigación-acción: conocer y cambiar la práctica educativa ( $\left.4^{\mathrm{a}} \mathrm{Ed}\right)$, Barcelona, Editorial Graó.

Leal Filho, W. (ed.). (2009), Sustainability at universitiesopportunities, challenges and trends, Frankfurt, Peter Lang.

Lozano, R. (2006), "Incorporation and institutionalization of SD into universities: breaking through barriers to change", Gournal of Cleaner Production, núm 14, pp. 787796.

Lozano, R., R. Lukman, F. J. Lozano, D. Huisinghd y W. Lambrechts (2013), "Declarations for sustainability in higher education: becoming better leaders, through addressing the university system", fournal of Cleaner Production, núm. 48, pp. 10-19.

Marsick, V. J. y T. E. Maltbia (2009), "The transformative potential of action learning conversations", en Transformative learning in practice: insights from community, workplace and higher education, San Francisco, Jossey-Bass, pp. 160-171.

McGill, I. y A. Brockbank (2004), The Action Learning Handbook: powerful techniques for education, professional development and training, Abingdon, Routledge Falmer.

McNiff, J. y J. Whitehead (2010), You and your action research project ( $3^{\mathrm{a}}$ ed.), Abingdon, Routledge.
Moore, J. (2005), "Barriers and pathways to creating sustainability education programs: policy, rhetoric and reality", Environmental Education Research, vol. 11, núm. 5, pp. 537-555.

Mora Penagos, W. M. (2012), "Ambientalización curricular en la educación superior: un estudio cualitativo de las ideas del profesorado", Profesorado. Revista de Currículum y Formación del Profesorado, vol. 16, núm. 2, pp. 77-103.

Müller-Christa, G., S. Sterling, R. van Dam-Mierasc, M. Adomßent, D. Fischerd, y M. Rieckmann (2014), "The role of campus, curriculum, and community in higher education for sustainable development-a conference report", Fournal of Cleaner Production, vol. 62, núm. 1, pp. 134-137.

Murga Menoyo, M. A. (2009), "La década de la educación para el desarrollo sostenible. Antecedentes y significado", Bordón, Revista de Pedagogía, vol. 61, núm. 2, pp. 93-103.

Murga-Menoyo, M. A. (2015), "Competencias para el desarrollo sostenible: las capacidades, actitudes y valores meta de la educación en el marco de la Agenda global post- 2015”, Foro de Educación, núm. 2, pp. 55-83.

Rapley, T. (2011), "Some pragmatics of data analysis", en Qualitative Research, Londres, SAGE Publications.

Reason, P. y H. Bradbury (2008), "Introduction", en The SAGE Handbook of Action Research: Participative Inquiry and Practice, Londres, SAGE Publications Ltd, pp. 1-10.

Revans, R. (2011), ABC of Action Learning, Farnham, Gower Publishing Limited.

Ryan, A. y D. Cotton (2013), "Times of change: shifting pedagogy and curricula for future sustainability", The Sustainable University: Progress and Prospects, Abingdon, Routledge, pp. 151-167.

Senge, P. (2006), The fifth discipline: the art E practice of the learning organisation ( $2^{\mathrm{a}}$ ed.), Londres, Random House Business Books.

Sipos, Y., B. Battisti y K. Grimm (2008), "Achieving transformative sustainability learning: engaging head, hands and heart", International fournal of Sustainability in Higher Education, vol. 9, núm. 1, pp. 68-86. 
Solís-Espallargas, C. y R. Valderrama-Hernández (2015), "La educación para la sostenibilidad en la formación de profesorado. ¿Qué estamos haciendo?”, Foro de Educación, núm. 19, pp. 165-192.

Somekh, B. (2006), Action research. A methodology for change and development, Buckingham, Open University Press.

Sterling, S. (2004), "Higher education, sustainability, and the role of systemic learning", Higher education and the challenge of sustainability: problematics, promise and practice, Dordrecht, Kluwer Academic Publishers, pp. 49-70.

Thomas, I. (2004), "Sustainability in tertiary curricula: what is stopping it happening?", International fournal of Sustainability in Higher Education, vol. 5, núm. 1, pp. 33-47.

Thomas, I. (2009), "Critical thinking, transformative learning, sustainable education, and problem-based learning in universities", Fournal of Transformative Education, vol. 7, núm. 3, pp. 245-264.

Tilbury, D. (2007), "Learning based change for sustainability: perspectives and pathways", en Social learning towards a sustainable world, Wageningen, Wageningen Academic Publishers, pp. 117-131.

Tilbury, D. (2012), "Higher education for sustainability: a global overview of commitment and progress", en Higher education in the world 4. Higher Education's Commitment to Sustainability: from understanding to action, Barcelona, Global University Network for Innovation, pp. 18-28.

Tilbury, D. y D. Wortman (2004), Engaging people in sustainability, Gland, IUCN.

Velazquez, L., N. Munguia, A. Platt y J. Taddei (2006), "Sustainable university: what can be the matter?", Fournal of Cleaner Production, núm. 14, pp. 810-819.

Vilches Peña, A., y D. Gil Pérez (2015), “Ciencia de la sostenibilidad: ¿una nueva disciplina o un nuevo enfoque para todas las disciplinas?", Revista Iberoamericana de Educación, vol. 69, núm. 1, pp. 39-60.

Wals, A. E. J. (2010), "Mirroring, gestaltswitching and transformative social learning. Stepping stones for developing sustainability competence", International Fournal of Sustainability in Higher Education, vol. 11, núm. 4, pp. 380-390.

Wiek, A., L. Withycombe y C. L. Redman (2011), "Key competencies in sustainability: a reference framework for academic program development", Sustainability Science, núm. 6, pp. 203-218.

Wright, T. S. A. (2010), "University presidents' conceptualizations of sustainability in higher education", International fournal of Sustainability in Higher Education, vol. 11, núm. 1, pp. 61-73.

\section{Cómo citar este artículo:}

Cebrián, Gisela (2020), "La educación para el desarrollo sostenible en el currículum universitario: una investigación-acción cooperativa con profesorado", Revista Iberoamericana de Educación Superior (RIES), vol. XI, núm. 30, pp. 99-114, https:// doi.org/10.22201/iisue.20072872e.2020.30.590 [Consulta: fecha de última consulta]. 